\title{
Ultrathin silicon solar cells with enhanced photocurrents assisted by plasmonic nanostructures
}

\author{
Xiao, Sanshui ; Stassen, Erik; Mortensen, N. Asger
}

Published in:

Journal of Nanophotonics

Link to article, DOI:

10.1117/1.JNP.6.061503

Publication date:

2012

Document Version

Publisher's PDF, also known as Version of record

Link back to DTU Orbit

Citation (APA):

Xiao, S., Stassen, E., \& Mortensen, N. A. (2012). Ultrathin silicon solar cells with enhanced photocurrents assisted by plasmonic nanostructures. Journal of Nanophotonics, 6(1), [061503].

https://doi.org/10.1117/1.JNP.6.061503

\section{General rights}

Copyright and moral rights for the publications made accessible in the public portal are retained by the authors and/or other copyright owners and it is a condition of accessing publications that users recognise and abide by the legal requirements associated with these rights.

- Users may download and print one copy of any publication from the public portal for the purpose of private study or research.

- You may not further distribute the material or use it for any profit-making activity or commercial gain

- You may freely distribute the URL identifying the publication in the public portal

If you believe that this document breaches copyright please contact us providing details, and we will remove access to the work immediately and investigate your claim. 


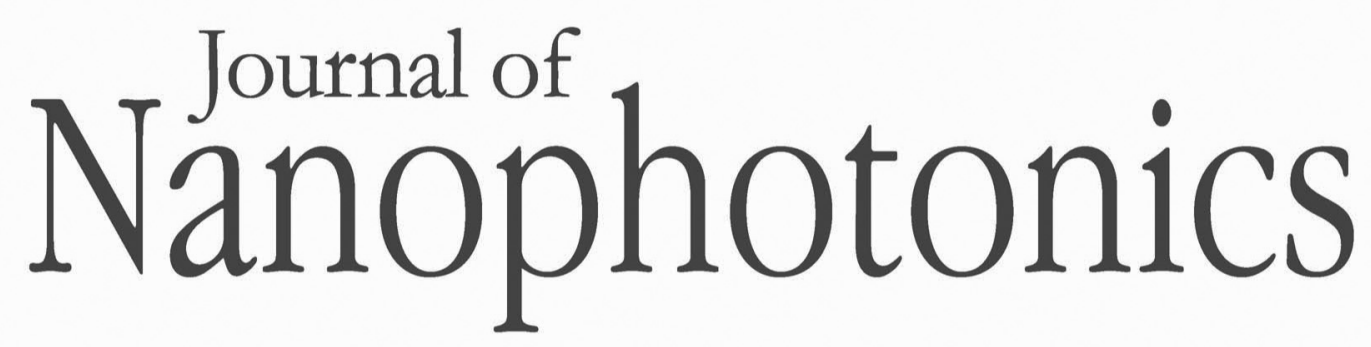

\section{Ultrathin silicon solar cells with enhanced photocurrents assisted by plasmonic nanostructures}

Sanshui Xiao

Erik Stassen

N. Asger Mortensen 


\title{
Ultrathin silicon solar cells with enhanced photocurrents assisted by plasmonic nanostructures
}

\author{
Sanshui Xiao, Erik Stassen, and N. Asger Mortensen \\ Technical University of Denmark, DTU-Fotonik, Department of Photonics Engineering, \\ DK-2800 Kongens Lyngby, Denmark \\ saxi@fotonik.dtu.dk
}

\begin{abstract}
Thin-film photovoltaics offers the potential for a significant cost reduction compared to traditional photovoltaics. However, the performance of thin-film solar cells is limited by poor light absorption. We have devised an ultra-thin-film silicon solar cell configuration assisted by plasmonic nanostructures. By placing a one-dimensional plasmonic nanograting on the bottom of the solar cell, the generated photocurrent for a $200 \mathrm{~nm}$-thickness crystalline silicon solar cell can be enhanced by $90 \%$ in the considered wavelength range, while keeping insensitive to the incident angle. These results are paving a promising way for the realization of high-efficiency thin-film solar cells. @ 2012 Society of Photo-Optical Instrumentation Engineers (SPIE). [DOI: 10.1117/ 1.JNP.6.061503]
\end{abstract}

Keywords: solar cells; plasmonics; absorption enhancement; grating.

Paper 11126SSP received Nov. 14, 2011; revised manuscript received Jan. 10, 2012; accepted for publication Jan. 12, 2012; published online Mar. 12, 2012.

\section{Introduction}

One of the greatest challenges facing our society today is the supply of low-cost, environmentally friendly energy sources that can meet the growing demands of population and economic growth. ${ }^{1}$ Photovoltaics, the conversion of sunlight to electricity, is already among the most efficient technologies for harvesting solar energy. Nowadays, bringing photovoltaics to the market is mainly limited by the high cost of electricity produced by the photovoltaic solar cell. To reduce the cost, thin-film solar cells are promising alternatives, but a limitation in thin-film solar cell technologies is that the absorbance of light is quite weak. The pyramidal surface texture technology has been used for trapping light for conventional solar cells. ${ }^{2,3}$ This kind of surface texture generally has a large surface area, leading to a high minority carrier recombination near the surface region of the thin-film solar cells. Recently, a new technology for achieving light trapping in thin-film solar cells by use of metallic nanostructures that support surface plasmons ${ }^{4-6}$ has been proposed. Light can be effectively trapped into a thin-film by engineering of metallic nanostructures, thereby increasing the absorption. ${ }^{7-15}$ Experimentally, improved photocurrent generation has been verified by placing or scattering metallic nanoparticles on the top of, within, or on the bottom of photovoltaic devices. ${ }^{16-18}$

Driven by new advanced nanofabrication technologies and the growing understanding of optical properties provided by plasmonic nanostructures, the use of periodic metallic nanostructures for photovoltaic applications has been received tremendous attention. Recently, placing one-dimensional periodic metallic nanostructures on the top of the thin-film solar cell is designed to realize broadband absorption enhancement. ${ }^{10,12}$ Since the excitation of surface plasmon polaritons is normally polarization sensitive, the design by the use of the one-dimensional metallic grating placed directly on the top of the solar cell will of course block a fairly large amount of total incident solar power. In this paper, we propose an ultra-thin-film solar cell, where absorption of the light in the active silicon layer is enhanced by placing one-dimensional metallic nanogratings at the bottom of the thin-film solar cell. Large integrated photocurrent enhancement for the wavelength of our interest is observed, which is attributed to the excitation of the hybrid

0091-3286/2012/\$25.00 (C) 2012 SPIE 
mode of the surface plasmon polaritons and the waveguide mode of the silicon thin-film. As a comparison, an ultrathin silicon solar cell with the metallic grating on the top of it is also investigated.

\section{Method for Evaluating the Generated Photocurrent of the Solar Cell}

Figure 1 depicts a thin-film solar cell configuration. The thin silicon layer acts as the device layer, where the absorption of the light is enhanced by plasmonic nanostructures. Due to a large surface area when introducing an array of plasmonic nanostructures, the silicon oxide layer is needed to provide excellent electrical surface passivation of silicon with high optical transparency. Here, we choose crystalline silicon as the active semiconductor material, which has a band-gap of $1.1 \mathrm{eV}$. If a photon is absorbed it has the possibility of exciting an electron from the valence band to the conduction band. Only if the photon has enough energy will the electron be excited into the conduction band from the valence band. Therefore, for photovoltaics application, we only consider the working wavelength below $1100 \mathrm{~nm}$, corresponding to the band-gap of the crystalline silicon.

In order to evaluate the solar cell system, we perform full-wave electromagnetic simulations with the aid of commercial simulation packages ${ }^{19}$ to determine the photocurrent enhancement compared to the reference structure consisting of the ultrathin crystalline silicon layer without metallic nanostructures. Broadband illumination in the wavelength range of 400 to $1100 \mathrm{~nm}$ is considered, and then the results are weighted by the AM 1.5G solar spectrum. We calculate the short circuit current per unit area, $J_{\mathrm{sc}}$, by

$$
J_{\mathrm{sc}}=\frac{q}{A} \int \Gamma_{\mathrm{GR}} d V
$$

where $q$ is the charge of an electron, $A$ is the illuminated area, $\Gamma_{\mathrm{GR}}$ is the electron generation rate per unit volume and the integration is over the volume containing generations. With the assumption that each photon absorbed within the active layer creates one electron-hole pair, the electron generation rate per unit volume can therefore be described by

$$
\Gamma_{\mathrm{GR}}=\int \operatorname{AM}(\omega)\left[\frac{\varepsilon^{\prime \prime}(\omega)|E(\omega)|^{2}}{2 \hbar}\right] d \omega,
$$

where $\operatorname{AM}(\omega)$ is the $\operatorname{AM} 1.5 \mathrm{G}$ solar spectrum, $|E(\omega)|^{2}$ is the magnitude of the electric field squared within the active layer, and $\varepsilon^{\prime \prime}(\omega)$ is the imaginary part of the dielectric function of the silicon $\left[\varepsilon(\omega)=\varepsilon^{\prime}(\omega)+j \varepsilon^{\prime \prime}(\omega)\right]$, which is obtained by a fit to the experimental data of Palik. ${ }^{20}$ We thus implicitly assume band-gap absorption to be the dominating phenomenon, represented by $\varepsilon^{\prime \prime}(\omega)$. Note that only the absorption occurring in the active layer contributes to the generated carrier, which should be separated from the absorption within the metal, resulting in Ohmic loss. Since the incident light from sun is unpolarized, we need to consider two

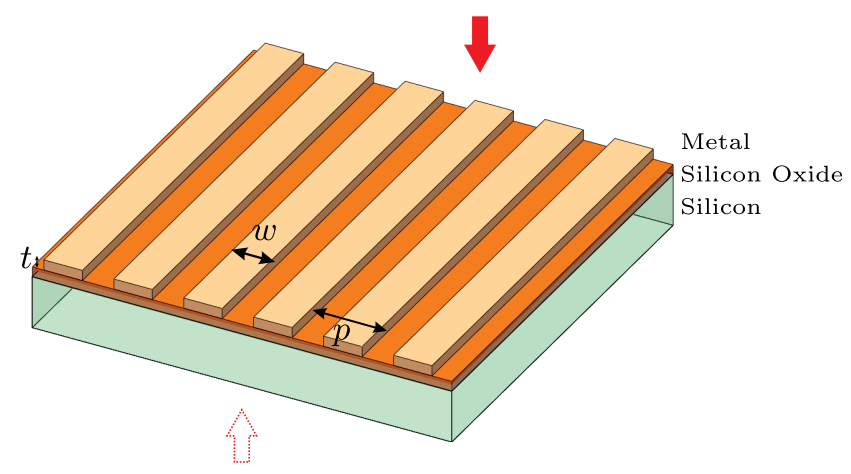

Fig. 1 Schematic of a thin-film solar cell utilizing a one-dimensional metallic nanograting. The structure may be subjected to either front-side or back-side illumination. 
polarizations: the TE polarization (the electric field polarized parallel to the grating) and the TM polarization (the magnetic field polarized parallel to the grating) and to take equal contribution from each polarization into account when evaluating the short circuit current.

\section{Ultra-thin-Film Solar Cells by Incorporating the Metallic Nanostructures}

Let us first consider an ultra-thin-film crystalline silicon solar cell by incorporating a onedimensional metallic grating on the top of it (see Fig. 1). The thickness of the crystalline silicon layer and the passivation layer are fixed as 200 and $10 \mathrm{~nm}$, respectively. The one-dimensional array of metallic strips is described by the period $p$, the thickness of the metallic strips $t$, and the width of strips $w$, respectively. Figure 2 shows the enhancement of the photocurrent per unit area of the thin-film solar cell when covering the metallic grating, normalized to the same structure without the metallic grating. The results illustrate how the photocurrent enhancement varies when tuning the width of the metal strip and the thickness of the metal grating. The period of the grating is chosen as $450 \mathrm{~nm}$. For the case of the TE polarization, the enhancement of the photocurrent shown in Fig. 2(a) is always below unity. In this case, the metallic grating generally acts as a reflection mirror, leading to higher reflection. Since the short circuit current is proportional to the absorbance of light in the thin-film layer, the enhancement factor becomes less than unity due to higher reflection by the metallic grating. With the decrease of the width of the metallic strips, the enhancement factor increases since less metallic material is obscuring transfer of light to the silicon layer. The enhancement factor for the photocurrent is not too sensitive to the thickness of the metal film when the thickness of the metal film is larger than $50 \mathrm{~nm}$. For the case of the TM polarization, due to the excitation of the plasmon resonance, i.e., a collective oscillation of the conduction electrons in the metal, the metallic strips with a size well below the wavelength of light can be used as subwavelength scattering elements to couple and trap freely propagating plane waves into the absorbing semiconductor thin-film by multiple and high-angle scattering. This is causing an increase in the effective optical path length in the thin-film solar cell. ${ }^{5}$ The enhancement factor for the TM polarization is shown in Fig. 2(b), where the values are larger than unity attributed to the metallic grating. The absorption of light is further enhanced when decreasing the width of the metallic strips and the enhancement factor reaches 1.4 for the case of $t=60 \mathrm{~nm}$ and $w=100 \mathrm{~nm}$. However, if we check the overall contribution from the metallic gratings, shown in Fig. 2(c), the total enhancement factor, defined by the average of the enhancement factors for two polarizations, is only about 1.2. One can conclude that the thin-film solar cell could benefit from the metallic nano-structure placed on the top of it. Due to the polarization dependence, the overall contribution arising from the metallic grating is not too high as expected. It should be pointed out that our conclusion is based on the thin-film solar cell assisted by a one-dimensional periodic metallic nanostructure. Two-dimensional periodic metallic nanostructures may lead to other conclusions.
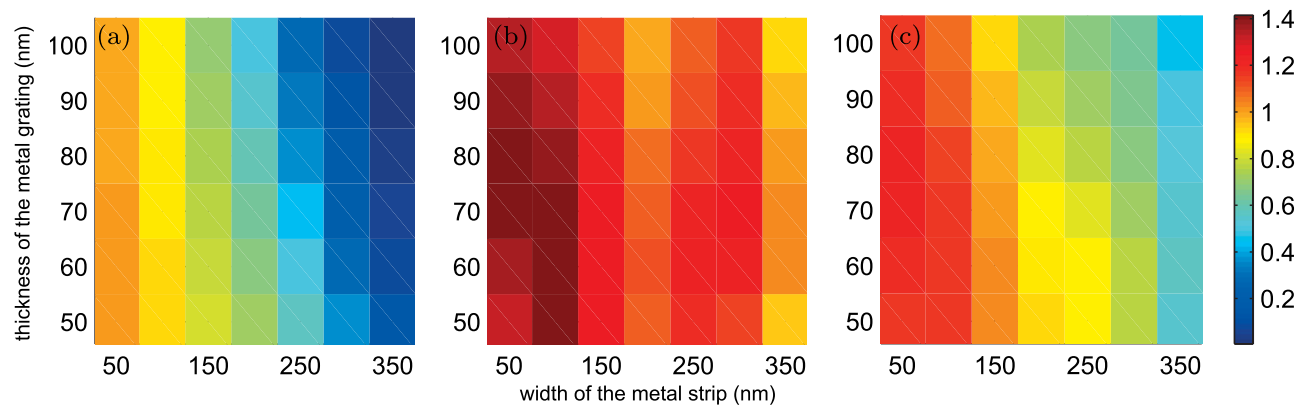

Fig. 2 Photocurrent enhancement for the ultrathin silicon solar cell assisted by the front-side metallic grating as a function the metal thickness and metallic strip width for the (a) TE, (b) TM, and (c) TE + TM polarization. The period is fixed as $450 \mathrm{~nm}$ and the light is normally incident with a broadband range from 400 to $1100 \mathrm{~nm}$. The enhancement factor is normalized with a reference to the result for the thin-film structure without any metallic nanostructures. 
It has been shown that the absorption of light in the thin-film solar cell can be enhanced by effective coupling to waveguide modes supported by the thin-film. ${ }^{9,10}$ Now, let us consider an ultra-thin-film solar cell assisted by the metallic grating placed on the bottom of it. Results for the photocurrent enhancement are shown in Fig. 3. The period of the grating is fixed to $450 \mathrm{~nm}$, while we are varying the width of the metal strips and the thickness of the metal film. It can be seen in Figs. 3(a) and 3(b) that the enhancement factors of the short circuit current for both two polarizations are significantly enlarged. The enhancement factor is strongly sensitive to the width of the metal strips, and slightly dependent on the thickness of the metal film. For the case of the TE polarization, the enhancement factor reaches a maximum value of 1.98 when $t=100 \mathrm{~nm}$ and $w=250 \mathrm{~nm}$. The maximum value of the enhancement factor for the TM polarization is around 2.04 when $t=50 \mathrm{~nm} \mathrm{~nm}$ and $w=300 \mathrm{~nm}$. By averaging the contributions from both the TE and TM polarizations, the total enhancement factor for the photocurrent shown in Fig. 3(c) is significantly improved, compared to the result for the previous case as shown in Fig. 2(c). For example, when $t=50 \mathrm{~nm}$ and $w=300 \mathrm{~nm}$, the total integrated photocurrent improvement under AM1.5G solar illumination can reach a factor of 1.82. For comparison, the maximum value for the enhancement factor for the solar cell assisted by the front-side metallic grating is only 1.2 .

To better understand the mechanism behind the enhanced photocurrent, here we plot absorption enhancement (the absorption of the light is governed by $\left[\varepsilon^{\prime \prime}(\omega) \omega|E(\omega)|^{2} / 2\right]$ ) as a function of wavelength, as well as the field patterns for some specific frequencies, as shown in Fig. 4. The absorption enhancement for the ultra-thin-film solar cell assisted by the metallic grating placed on the bottom of the solar cell is shown as the solid line when $t=100 \mathrm{~nm}, w=250 \mathrm{~nm}$, and $p=450 \mathrm{~nm}$ for the case of the TE polarization and the dashed line is for the TM polarization when $t=50 \mathrm{~nm}, w=300 \mathrm{~nm}$, and $p=450 \mathrm{~nm}$. It turns out that for the wavelength of our interest the absorption enhancement is always larger than unity, leading to a high total enhancement factor of the short circuit current. Field patterns for two main peaks $(\lambda=632$ and $871 \mathrm{~nm})$ with respect to the normalized field $\left(H / H_{0}\right)$ for the TE polarization are shown in the left-hand side of the two peaks, where $H_{0}$ is the magnetic field of the incident wave. The value of the normalized field, with a maximum value of 9 , is strongly enhanced by adding the metallic nanostructure. We believe that the enhancement is attributed to strong backscattering by the metallic grating. For the normalized field pattern $\left(E / E_{0}\right)$ for two main peaks $(\lambda=680$ and $932 \mathrm{~nm})$ of the TM polarization ( $E_{0}$ is the electric field of the incident wave), shown in the right-hand side of the two peaks, it can be clearly seen that the surface plasmon polaritons near the silicon and metal interface are excited and that the normalized field has a maximum value close to 11 . We believe that for the TM polarization the absorption enhancement is arising from the excitation of the hybrid mode of the surface plasmon polaritons and the waveguide mode of the silicon thin-film.

The results shown above are all associated with the solar cell when fixing the periodicity and the incident angle. It is naturally interesting to investigate the influence of the period and incident angle to the photocurrent enhancement. Figure 5(a) shows the influence of the periodicity to the enhancement factor of the short circuit current when $t=50 \mathrm{~nm}$ and
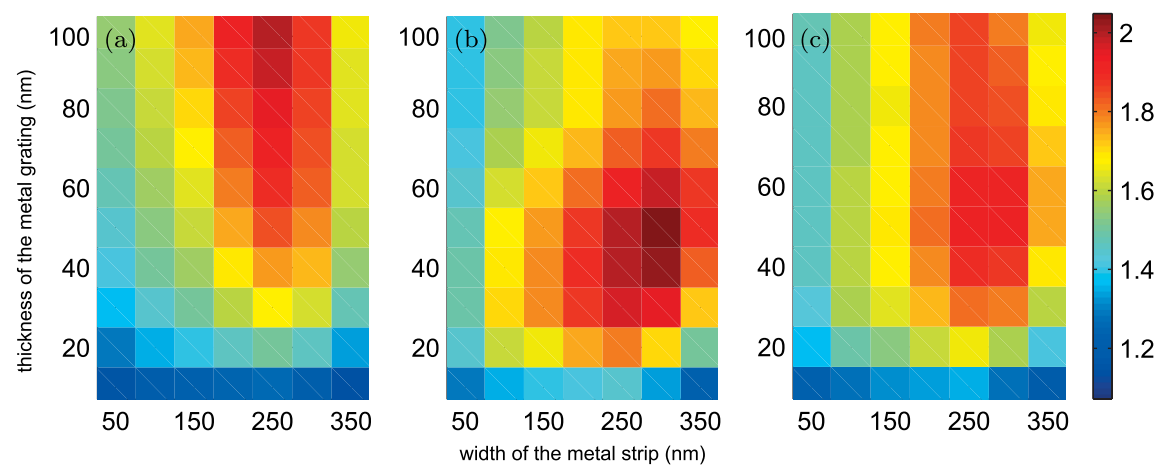

Fig. 3 Photocurrent enhancement for the ultrathin silicon solar cell assisted by the back-side metallic grating as a function the metal thickness and metallic strip width for the (a) TE, (b) TM, and (c) TE + TM polarization. 
Xiao, Stassen, and Mortensen: Ultrathin silicon solar cells with enhanced photocurrents...

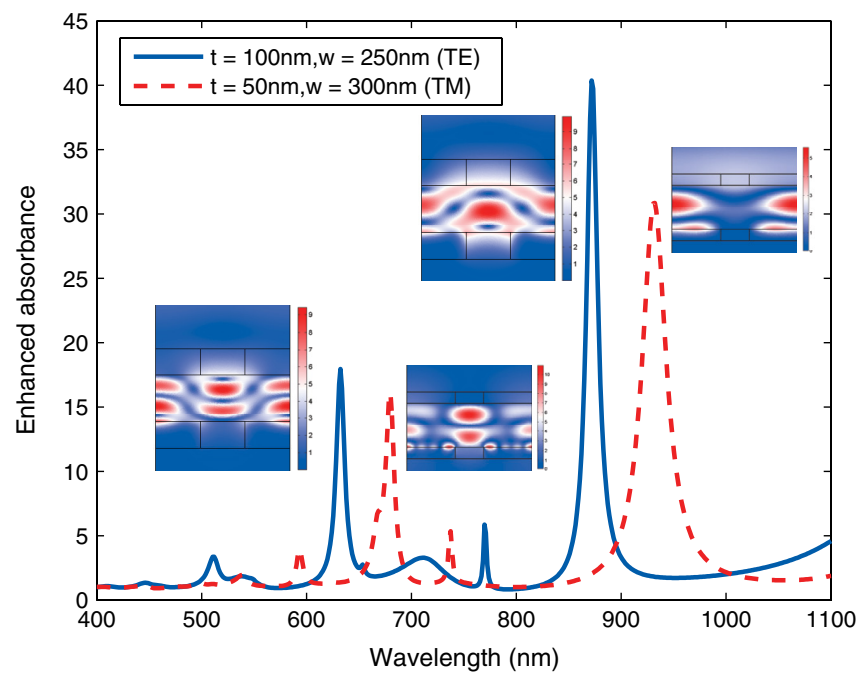

Fig. 4 Absorption enhancement for the ultra-thin-film solar cell assisted by the back-side metallic grating for the case of the TE polarization (the solid line) when $t=100 \mathrm{~nm}, w=250 \mathrm{~nm}$, and $p=$ $450 \mathrm{~nm}$ and the TM polarization case (the dashed line) when $t=50 \mathrm{~nm}, w=300 \mathrm{~nm}$, and $p=450 \mathrm{~nm}$. Insets show the normalized field patterns at the resonant positions.
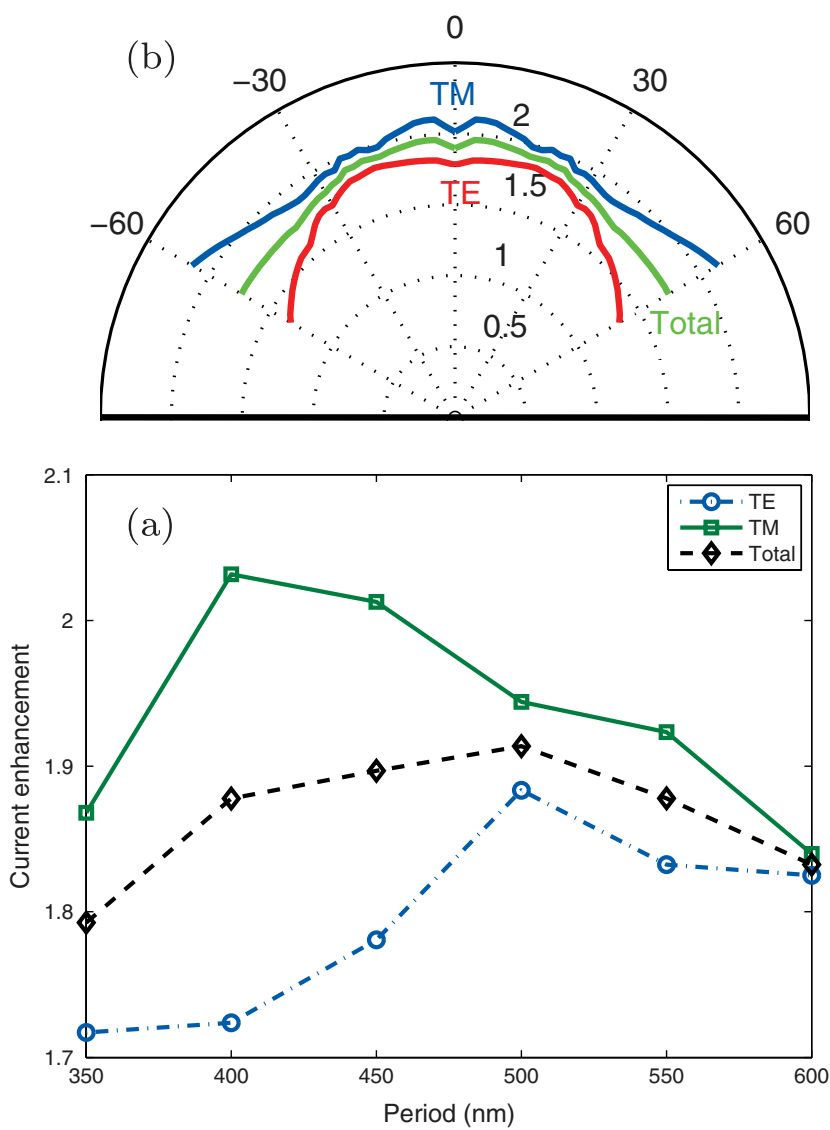

Fig. 5 (a) Photocurrent enhancement as a function of the incident angle, when $p=450 \mathrm{~nm}$, $w=50 \mathrm{~nm}$, and $t=50 \mathrm{~nm}$. (b) Photocurrent enhancement as a function of the periodicity when $w=300 \mathrm{~nm}$ and $t=50 \mathrm{~nm}$ for the normal incidence. 
$w=300 \mathrm{~nm}$. The photocurrent enhancement is dependent on the period of the metallic grating. Figure 5(a) illustrates that the enhancement under AM1.5G solar illumination can reach a factor of 1.9 when $p=500 \mathrm{~nm}$. To the best of our knowledge, it is the simplest thin-film silicon solar cell configuration which provides the best performance in terms of the enhanced generation photocurrent. When placing the metallic grating on top of the solar cell, it has been shown that the enhancement of the short circuit current is quite sensitive to the incident angle. ${ }^{12}$ For the case of the thin-film solar cell assisted by the back-side metallic grating, influence of the incident angle to the enhanced photocurrent is shown in Fig. 5(b). The photocurrent enhancement for the TE polarization is relatively sensitive to the incident angle, while the total enhancement factor under an unpolarized illumination is almost immune to the incident angle ranging from $0 \mathrm{deg}$ to $60 \mathrm{deg}$.

\section{Conclusions and Discussions}

In this paper, we have proposed an ultra-thin-film solar cell improved by the presence of plasmonic nanostructures. The solar cells assisted by the front-side metallic and the back-side metallic gratings have been investigated. Integrated photocurrent enhancement in the thin-film silicon solar cell has been evaluated, and results show that large photocurrent enhancement with a factor of 1.9 is achieved by placing a one-dimensional metallic grating on the bottom of the solar cell, while being insensitive to the incident angle. The results presented here could be used for the realization of low-cost and high-efficiency thin-film solar cells. Here, we have deliberately focused on metallic nanostructures. Recent work suggests that dielectric nanostructures may be at least equally useful for energy harvesting. For future work it would be interesting to explore opportunities offered by combination of such ideas.

\section{Acknowledgments}

We thank Prof. Jesper Mørk, Prof. Ole Hansen, and Prof. Ole Sigmund for stimulating discussions. This work is partly supported by the Catalysis for Sustainable Energy Initiative Center, funded by the Danish Ministry of Science, Technology and Innovation.

\section{References}

1. N. S. Lewis and D. G. Nocera, "Powering the planet: chemical challenges in solar energy ultilization," PNAS 103(43), 15729-15735 (2006).

2. E. Yablonovitch and G. D. Cody, "Intensity enhancement in textured optical sheets for solar cells," IEEE Trans. Elect. Dev. 29(2), 300-305 (1982).

3. H. W. Deckman, C. B. Roxlo, and E. Yablonovitch, "Maximum statistical increase of optical absorption in textured semiconductor films," Opt. Lett. 8(9), 491-493 (1983).

4. H. Raether, Surface Plasmons on Smooth and Rough Surfaces and on Gratings, Springer, Berlin (1988).

5. K. R. Catchpole and A. Polman, "Plasmonic solar cells," Opt. Express 16(26), 21793-21800 (2008).

6. H. A. Atwater and A. Polman, "Plasmonics for improved photovoltaic devices," Nat. Mater. 9, 205-213 (2010).

7. S. Xiao and M. Qiu, "Theoretical study of the transmission properties of a metallic film with surface corrugations," J. Opt. A: Pure Appl. Opt. 9, 348-351 (2007).

8. Z. Yu, A. Raman, and S. Fan, "Fundamental limit of nanophotonic light trapping in solar cells," PNAS 107, 17491-17496 (2010).

9. V. E. Ferry et al., "Plasmonic nanostructure design for efficient light coupling into solar cell," Nano Lett. 8(12), 4391-4397 (2008).

10. R. A. Pala et al., "Design of plasmonic thin-film solar cells with broadband absorption enhancements," Adv. Mater. 21, 3504-3509 (2009).

11. S. Xiao et al., "Nealy-zero transmission through periodically modulated ultrathin metal films," Appl. Phys. Lett. 97, 071116 (2010). 
12. J. N. Munday and H. A. Atwater, "Large integrated absorption enhancement in plasmonic solar cells by combining metallic gratings and antireflection coatings," Nano Lett. 11(6), 2195-2201 (2011).

13. K. Soderstrom et al., "Photocurrent increase in n-i-p thin film silicon solar cells by guided mode excitation via grating coupler," Appl. Phys. Lett. 96, 213508 (2010).

14. V. E. Ferry, J. N. Munday, and H. A. Atwater, "Design considerations for plasmonic photovoltaics," Adv. Mater. 22, 4794-4808 (2010).

15. S. Xiao and N. A. Mortensen, "Surface-plasmon-polariton-induced suppressed transmission through ultrathin metal disk arrays," Opt. Lett. 36(1), 37-39 (2011).

16. B. P. Rand, P. Peumans, and S. R. Forrest, "Long-range absorption enhancement in organic tandem thin-film solar cells containing silver nanoclusters," J. Appl. Phys. 96(12), 7519-7526 (2004).

17. D. M. Schaadt, B. Feng, and E. T. Yu, "Enhanced semiconductor optical absorption via surface plasmon excitation in metal nanoparticles," Appl. Phys. Lett. 86, 063106 (2005).

18. K. Nakayama, K. Tanabe, and H. A. Atwater, "Plasmonic nanoparticle enhanced light absorption in gaas solar cells," Appl. Phys. Lett. 93, 121904 (2008).

19. COMSOL Multiphysics, COMSOL AB, Sweden.

20. E. D. Palik, Handbook of Optical Constants of Solids, Academic, New York (1985).

Biographies and photographs of the authors are not available. 